\title{
Standard Comorbidity Measures Do Not Predict Patient-reported Outcomes 1 Year After Total Hip Arthroplasty
}

\author{
Meridith E. Greene BA, Ola Rolfson MD, PhD, \\ Max Gordon MD, PhD, Göran Garellick MD, PhD, \\ Szilard Nemes PhD
}

Published online: 21 February 2015

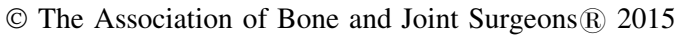

\begin{abstract}
Background Comorbidities influence surgical outcomes and therefore need to be included in risk adjustment when predicting patient-reported outcomes. However, there is no consensus on how best to use the available data about comorbidities in registry-based predictive models.

Questions/purposes The purposes of this study were (1) to determine whether the International Classification of Diseases, $10^{\text {th }}$ Revision (ICD-10)-based comorbidity
\end{abstract}

This work is funded by the Swedish Hip Arthroplasty Register. One author (OR) has received funding from grants from Doktor Felix Neuberghs Foundation, the Göran Bauer Grant, The Swedish Society of Medicine, and The Wangstedts Foundation.

All ICMJE Conflict of Interest Forms for authors and Clinical Orthopaedics and Related Research ${ }^{\mathbb{R}}$ editors and board members are on file with the publication and can be viewed on request.

Each author certifies that his or her institution approved the human protocol for this investigation, that all investigations were conducted in conformity with ethical principles of research, and that informed consent for participation in the study was obtained.

The majority of the work for this study was conducted at the Swedish Hip Arthroplasty Register, Gothenburg, Sweden.

M. E. Greene ( $₫)$, O. Rolfson

Harris Orthopaedic Laboratory, Massachusetts General Hospital, 55 Fruit Street, GRJ 1125, Boston, MA 02114, USA

e-mail: megreene@partners.org

M. E. Greene, O. Rolfson, M. Gordon, G. Garellick, S. Nemes Swedish Hip Arthroplasty Register, Gothenburg, Sweden

M. E. Greene, O. Rolfson, G. Garellick, S. Nemes Department of Orthopaedics, Institute of Clinical Sciences, Sahlgrenska Academy, University of Gothenburg, Gothenburg, Sweden

M. Gordon

Department of Clinical Sciences at Danderyd Hospital,

Karolinska Institute, Stockholm, Sweden measures (Elixhauser, Charlson, and Royal College of Surgeons Charlson) offer added value in explaining patients' health-related quality of life (HRQoL), pain, and satisfaction after total hip arthroplasty (THA) when preoperative HRQoL, pain, and Charnley classification were known; and (2) to determine the ideal timeframe for recording the different diagnoses that serves as the basis for comorbidity measure calculations.

Methods There were 22,263 patients who had undergone THA with complete pre- and postoperative patient-reported outcome measures (PROMs) included in the Swedish Hip Arthroplasty Register between 2002 and 2007. The three comorbidity indices were calculated with ICD-10 codes identified in the Swedish National Patient Register from 1, 2 , and 5 years before the patient underwent THA. The impact of the comorbidity indices on the PROM scores (EQ-5D index, EQ visual analog scale [VAS], pain VAS, and satisfaction VAS) was modeled with linear regression where the 1-year patient postoperative outcome score was the dependent variable and independent variables included patient preoperative Charnley classification, preoperative HRQoL and pain, and comorbidity indices. The partial $R^{2}$ value indicated how much each variable uniquely contributed to the predictive capacity of the model.

Results The ICD-10-based comorbidity measures added little predictive value to the models for each of the outcomes of interest (EQ-5D index, EQ VAS, pain VAS, and satisfaction VAS). Charnley classification and the preoperative scores were the strongest predictors of both measures of postoperative HRQoL, of postoperative pain, and postoperative satisfaction with outcomes from surgery. Of all the predictors considered, only the Charnley classification was associated with all outcomes, irrespective of the timeframe considered. For each of the outcomes considered, there was a gradual increase in the models' 
predictive power with the length of the timeframe considered for calculating the comorbidity measures.

Conclusions For predicting outcomes 1 year after THA, we found that there was no added value in ICD-10-based comorbidity measures if patient Charnley classification and preoperative HRQoL and pain measures were known. Level of Evidence Level III, therapeutic study.

\section{Introduction}

Assessment of achievement in orthopaedic surgery is shifting toward resulting patient health-related quality of life (HRQoL) rather than the measure of success or failure of joint implants as defined by crude endpoints like revision. Ahmad et al. [1] concluded that the ideal outcome measure for patients who have undergone THA should be specific for the hip, possess a generic component, and take into consideration patient comorbidities and the need for walking aids. Although no such ideal outcome measure exists, numerous patient-reported outcome measures (PROMs) appear in the literature and practitioners have augmented the measures with different comorbidity scores. PROMs not only help assess patient eligibility for elective surgery, but also can serve as predictors of patient quality of life after hip surgery [12]. Existing comorbidities are known to influence the outcome of surgery and, depending on the severity, can inhibit elective procedures such as THA. As a result, researchers and clinicians often consider comorbidity scores such as those determined using the Charlson Comorbidity Index, Charnley classification, and the American Society of Anesthesiologists physical function score, among others [2]. Comorbidities are defined as patient conditions or diseases not associated with the development or causation of the immediate disease of interest, and comorbidities can be diagnosed at different points in time, which may lead to different associations with the risk of adverse outcomes [18]. Thus, it is necessary to establish the best timeframe for calculating summary comorbidity measures.

Originally, the Swedish Hip Arthroplasty Register's PROMs program routinely collected data regarding patient pre- and postoperative $\mathrm{HRQoL}$ for the purpose of monitoring the performance of the nation's healthcare centers [22]. More recently, with healthcare centers achieving high success rates, the focus has been diverted to the goal of identifying patients who might not experience sufficient improvement in HRQoL. To this end, the Swedish Hip Arthroplasty Register proposed the construction of a decision-making support algorithm to estimate HRQoL 1 year postsurgery of patients who underwent THA. Such an instrument needed to consider both hospital- and patient- related factors. Existing patient comorbidities are known to influence the outcome of medical procedures, but there is no consensus on how best to use the available data about comorbidities in registry-based predictive models.

The objective of our study was to determine whether International Classification of Diseases, $10^{\text {th }}$ Revision (ICD-10)-based comorbidity measures (Elixhauser, Charlson, and the Royal College of Surgeons [RCS] Charlson) had added value for explaining postoperative HRQoL, pain, and satisfaction with the outcomes of surgery for patients who had undergone THA when preoperative HRQoL, pain, and Charnley classification were already known. We also aimed to investigate the ideal timeframe for recording the different diagnoses that served as the basis for comorbidity measure calculations.

\section{Materials and Methods}

We performed a registry study with prospectively collected PROM data from the national Swedish Hip Arthroplasty Register. These data were then merged with diagnosis data from the Swedish National Patient Register where each of the comorbidity scores was calculated retrospectively on the basis of patient medical diagnoses contained therein.

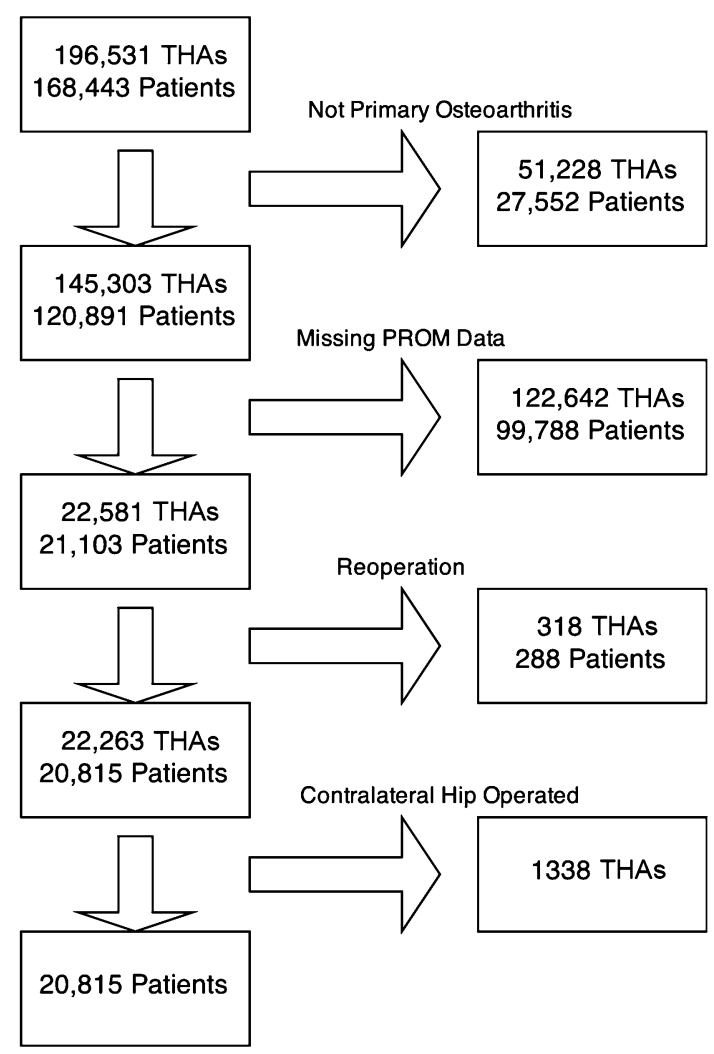

Fig. 1 This flowchart indicates patient selection from the Swedish Hip Arthroplasty Register. 
The Swedish Hip Arthroplasty Register data initially identified 22,263 patients who had undergone THA (Fig. 1). Mean patient age at time of surgery was 68 years ( \pm 9.9 years). The surgeries were performed between January 1, 2002, and December 28, 2007, with complete preoperative and postoperative PROM data available. If a patient had both hips operated on during the observation period, only the first hip with complete PROMs was included in the analysis. There was a slight predominance of women patients $(12,696)$. To calculate comorbidity scores for each patient, we obtained individual diagnoses (ICD-10) from the Swedish National Patient Register. The two registers were crossmatched with the help of personal identity numbers unique to each Swedish resident to establish demographic, surgical, PROM, and comorbidity data for each patient (Table 1). Data collection and merger of the two databases was approved by the national ethical review board.

Table 1. Summary of study population PROMs pre- and post-THA, Charnley classification,and ICD-10-based comorbidity measures

\begin{tabular}{|c|c|c|c|c|c|}
\hline \multirow[t]{2}{*}{ Scale } & \multicolumn{4}{|l|}{ Preoperative } & \multirow{2}{*}{$\begin{array}{l}\text { Postoperative } \\
1 \text { year }\end{array}$} \\
\hline & 5 years & 2 years & 1 year & Immediately & \\
\hline EQ-5D index, mean $\pm S D$ & & & & $0.41 \pm 0.31$ & $0.78 \pm 0.23$ \\
\hline $\mathrm{EQ}$ VAS, mean $\pm \mathrm{SD}$ & & & & $54.0 \pm 22.3$ & $75.8 \pm 19.9$ \\
\hline Pain VAS mean $\pm \mathrm{SD}$ & & & & $61.1 \pm 16.7$ & $14.2 \pm 17.8$ \\
\hline Satisfaction VAS, mean \pm SD & & & & - & $16.2 \pm 19.9$ \\
\hline \multicolumn{6}{|l|}{ Charnley classification, number $(\%)$} \\
\hline A & & & & $9263(45)$ & \\
\hline $\mathrm{B}$ & & & & $2757(13)$ & \\
\hline $\mathrm{C}$ & & & & $8795(42)$ & \\
\hline $\begin{array}{l}\text { Charlson index, mean } \pm \mathrm{SD} \\
\text { number }(\%)\end{array}$ & $0.27 \pm 0.60$ & $0.19 \pm 0.50$ & $0.15 \pm 0.44$ & & \\
\hline 0 & $17,558(79)$ & $18,773(84)$ & $19,425(87)$ & & \\
\hline 1 & $3632(16)$ & $2826(13)$ & $2383(11)$ & & \\
\hline 2 & $832(4)$ & $532(2)$ & $372(2)$ & & \\
\hline 3 & $179(0.8)$ & $102(0.5)$ & $63(0.3)$ & & \\
\hline 4 & $51(0.2)$ & $24(0.1)$ & $17(0.1)$ & & \\
\hline 5 & $10(0)$ & $5(0.0)$ & $3(0)$ & & \\
\hline 6 & $1(0)$ & $1(0.0)$ & $0(0)$ & & \\
\hline $\begin{array}{l}\text { RCS Charlson index, mean } \pm \text { SD } \\
\text { number }(\%)\end{array}$ & $0.12 \pm 0.39$ & $0.12 \pm 0.39$ & $0.12 \pm 0.39$ & & \\
\hline 0 & $19,925(90)$ & $19,941(90)$ & $19,953(90)$ & & \\
\hline 1 & $1978(9)$ & $1966(9)$ & $1957(9)$ & & \\
\hline 2 & $311(1)$ & 308 (1) & 305 (1.4) & & \\
\hline 3 & $45(0.2)$ & $44(0.2)$ & $44(0.2)$ & & \\
\hline 4 & $4(0)$ & $4(0)$ & $4(0)$ & & \\
\hline $\begin{array}{l}\text { Elixhauser index, mean } \pm \mathrm{SD} \\
\text { number }(\%)\end{array}$ & $0.59 \pm 0.94$ & $0.48 \pm 0.84$ & $0.42 \pm 0.78$ & & \\
\hline 0 & $13,989(63)$ & $15,265(69)$ & $15,962(72)$ & & \\
\hline 1 & 4973 (22) & $4403(20)$ & 4099 (18) & & \\
\hline 2 & $2223(10)$ & $1835(8)$ & $1618(7)$ & & \\
\hline 3 & 741 (3) & 539 (2) & $428(2)$ & & \\
\hline 4 & $229(1)$ & $162(0.7)$ & $114(0.5)$ & & \\
\hline 5 & $76(0.3)$ & $49(0.2)$ & $36(0.2)$ & & \\
\hline 6 & $23(0.1)$ & $8(0)$ & $4(0)$ & & \\
\hline 7 & $8(0)$ & $2(0)$ & $2(0)$ & & \\
\hline 8 & $1(0)$ & $0(0)$ & $0(0)$ & & \\
\hline
\end{tabular}

PROMs = patient-reported outcome measures; ICD-10 = International Classification of Diseases, 10th Revision; VAS = visual analog scale; RCS = Royal College of Surgeons. 


\section{Data Analyses}

We calculated the Elixhauser, Charlson, and the RCS Charlson Comorbidity Indices for each patient using ICD10 diagnosis codes based on the algorithms developed by Quan et al. [19]. (For further details, we refer the reader to Gordon and collaborators [11].) The three indices identified possible comorbidities and, based on their pathophysiologic properties, group comorbidities in different categories. The Charlson Comorbidity Index recognizes 19 distinct categories; the RCS Charlson Index has 14, whereas the Elixhauser has 31 distinct categories. If a patient had any illness identified, the different comorbidity categories (ie, the patient's medical history included the ICD-10 classification code during the studied time period), then that category was assigned a value of 1 ; if no ICD-10 codes appeared in the record, the value given was zero.

The RCS Charlson and Elixhauser comorbidity scores assume that each of the distinct categories weighs equally on patient health and the final index value was the sum of the different categorical values. Elixhauser et al. questioned this approach because the impact of disease categories may vary among different outcomes [8]; however, counting comorbidities was recently found to be a viable alternative to weighted indices [11]. The Charlson Index assigns weight for each category based on the seriousness of the disease group and the final index is a weighted sum of the different categorical values [19].

We calculated the Elixhauser, Charlson, and RCS Charlson comorbidity scores based on three overlapping timeframes. First, we went back 1 year from the actual surgery date and extracted all ICD-10 diagnosis codes that the patient had at that point. Then, we extended the presurgery timeframe to 2 years and then to 5 years before THA. Thus, for every patient, the 1-year comorbidity score should have been equal to or greater than the 2-year comorbidity score, which should have been equal to or greater than the score 5 years before THA.

The PROM protocol consisted of the HRQoL measure EQ-5D, a visual analog scale (VAS) for pain (pain is rated from 0 to 100 , where $0=$ no pain), the Charnley classification survey, and a VAS measuring patient satisfaction with the outcomes of surgery (from 0 to 100 , where $0=$ complete satisfaction) [3,9]. There are three Charnley classifications of musculoskeletal impairment: Class A, which corresponds to unilateral hip disease; Class B, which corresponds to bilateral hip disease; and Class $\mathrm{C}$, which indicates multiple joint disease or some other condition that inhibits the patient's ability to walk. The surveys were administered preoperatively (excluding the satisfaction VAS) and then 1 year after THA. The protocol was implemented in the Swedish Hip Arthroplasty Register in 2002 and gradually gained nationwide coverage [22].

The impact of the comorbidity scores on the four patient-reported outcomes (PROs) were modeled with linear regression (EQ-5D index, EQ VAS, pain VAS, and satisfaction VAS). For each of the three timeframes, we built a regression analysis that included each of the 1-year followup PROs as the dependent outcome. The 1-year timeframe model included patient preoperative Charnley class, the preoperative value of the considered PRO, and the comorbidity scores calculated on diagnoses reported 1 year before THA. The 2-year presurgery timeframe model replaced the comorbidity scores from the previous model with comorbidity scores calculated on patient diagnoses reported 2 years before THA. The 5-year, preTHA timeframe model replaced the patient comorbidity scores from the previous model with comorbidity scores calculated on diagnoses reported 5 years before THA.

To determine the influence of the three comorbidity measure on the PROs, the global predictive power of the models was summarized by the coefficient of determination $\left(\mathrm{R}^{2}\right)$, which denotes the proportion of variance in the outcome explained by the predictors. To understand the individual impact of each predictor, we assessed its partial $\mathrm{R}^{2}$ value to determine how much each variable uniquely contributed to the global $R^{2}$ value. To counteract possible distortion by including all three comorbidity indices in a single model, we calculated the partial $\mathrm{R}^{2}$ value for univariate models regressing the postoperative PROMs on each of the different comorbidity indices. These models were first adjusted for Charnley classification and then were adjusted for the appropriate preoperative PROM score.

To gauge if there was improvement in the predictive power with the use of a longer timeframe for calculating the comorbidity indices, we calculated the difference between the predictive power of the model based on patient 1-year comorbidity scores with the 2-year and 5-year comorbidity scores, respectively. By using 1000 nonparametric bootstraps, we built 95\% confidence intervals (CIs) of the differences between the $\mathrm{R}^{2}$ values. If the interval included zero, then we concluded that there was no evidence suggesting that a longer timeframe would increase the predictive possibilities of the model. In addition, we tested to see whether we could obtain an increase in precision if the interaction between the comorbidity indices and Charnley classification was included in the model. Although we saw an interaction between Charnley Class C and the comorbidity indices as one would expect, this was not accompanied by an increase in predictive power (data not shown), and so these interactions were not pursued further. 


\section{Results}

Independent of the length of followup, the models generally had low predictive power. Use of the Charnley classification, the three comorbidity scores, and the preoperative value of the outcome explained roughly $10 \%$ of the variation observed in EQ-5D index and EQ VAS; however, the pain VAS $(3 \%)$ and the satisfaction VAS (1\%) were insufficiently explained by the aforementioned predictors.

Predictive Value of Comorbidity Measures

Charnley classification and the preoperative scores were the strongest predictors of both measures of postoperative HRQoL (EQ-5D index and EQ VAS) and of postoperative pain (pain VAS). Similarly, Charnley classification was the strongest predictor for postoperative satisfaction with outcomes from surgery (satisfaction VAS). Regardless of the timeframe considered, the Charlson and Elixhauser comorbidity index scores had a marginal influence on the postoperative EQ-5D index $(B=-0.023 ; 95 \% \mathrm{CI},-0.035$ to $-0.011 ; \mathrm{p}<0.001 ;$ and $B=-0.010 ; 95 \% \mathrm{CI},-0.015$ to $-0.004 ; \mathrm{p}<0.001$, respectively) (Fig. 2). Similarly for the postoperative EQ VAS, the Charlson and Elixhauser comorbidity index scores had a marginal influence on the outcome score $(\beta=-3.407 ; 95 \% \mathrm{CI},-4.400$ to -2.414 ; $\mathrm{p}<0.001$; and $\beta=-0.886 ; 95 \% \mathrm{CI},-1.353$ to -0.420 ; $\mathrm{p}<0.001$, respectively) (Fig. 3). The pain VAS was not influenced by the Charlson or RCS Charlson scores, but was marginally influenced by the Elixhauser comorbidity

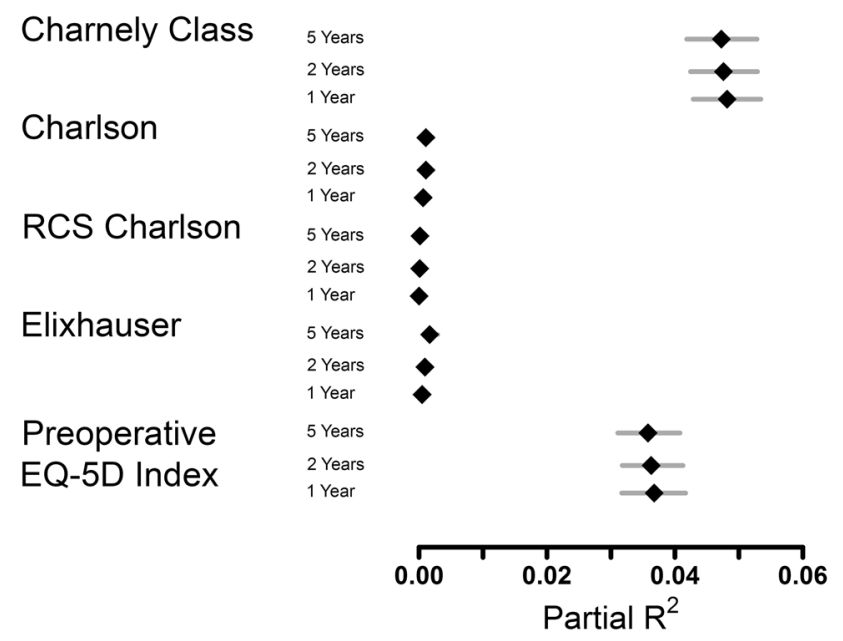

Fig. 2 This forest plot displays the predictive power of ICD-10-based comorbidity measures, self-reported disease-specific comorbidity measure, and the preoperative EQ-5D index values of the postoperative EQ-5D index values. index score $(B=0.522 ; 95 \% \mathrm{CI}, 0.091-0.954 ; \mathrm{p}=0.018)$ (Fig. 4). The Elixhauser comorbidity index was the only score of the three comorbidity measures to influence the satisfaction VAS at 1 year $(B=0.628 ; 95 \% \quad \mathrm{CI}$, 0.141-1.116; $\mathrm{p}=0.012$ ) (Fig. 5). Finally, the low predictive power of each of the comorbidity indices on the PROM values was not found to be the result of colinearity in the tested models (Table 2).

Ideal Timeframe for Diagnosis Recording

For each of the outcomes considered (EQ-5D index, EQ VAS, pain VAS, and satisfaction VAS), there was a gradual increase in the models' predictive power with the length of the timeframe considered for calculating the comorbidity measures. For the EQ-5D index, the predictive power of the 1-year timeframe was lower than the 2-year timeframe model $\left(\mathrm{R}^{2}\right.$ difference $=-0.003 ; 95 \% \mathrm{CI},-0.0049$ to $-0.0019 ; \mathrm{p}=0.001)$. Similarly, the 5-year timeframe model outperformed both the 1-year timeframe model $\left(\mathrm{R}^{2}\right.$ difference $=-0.006 ; 95 \% \quad \mathrm{CI},-0.0083$ to -0.0039 ; $\mathrm{p}=0.001)$ and the 2-year timeframe model $\left(\mathrm{R}^{2}\right.$ difference $=-0.002 ; 95 \% \mathrm{CI},-0.0046$ to $-0.0011 ; \mathrm{p}=0.013$ ) (Table 3). For the EQ VAS, the predictive power of the 1-year timeframe model was significantly lower than the 2-year timeframe model $\left(\mathrm{R}^{2}\right.$ difference $=-0.0031 ; 95 \%$ $\mathrm{CI},-0.0046$ to $-0.0019 ; \mathrm{p}=0.001)$. Similarly, the 5-year timeframe model outperformed both the 1-year timeframe model $\left(\mathrm{R}^{2}\right.$ difference $=-0.005 ; 95 \% \mathrm{CI},-0.0078$ to $-0.0038 ; \mathrm{p}=0.001)$ and the 2-year timeframe model $\left(\mathrm{R}^{2}\right.$ difference $=-0.002 ; 95 \% \mathrm{CI},-0.0045$ to -0.0011 ;

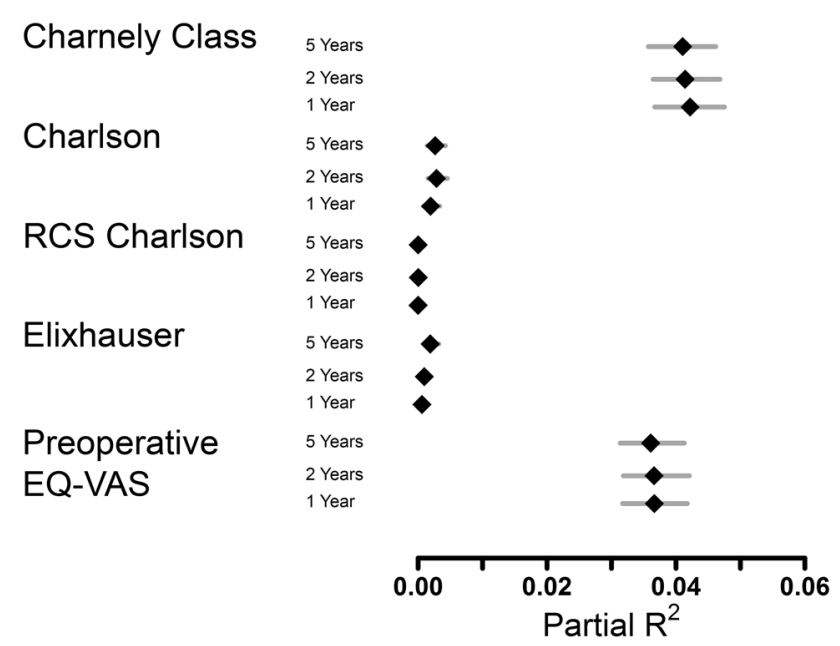

Fig. 3 This forest plot displays the predictive power of ICD-10-based comorbidity measures, self-reported disease-specific comorbidity measure, and the preoperative EQ VAS values of the postoperative EQ VAS values. 


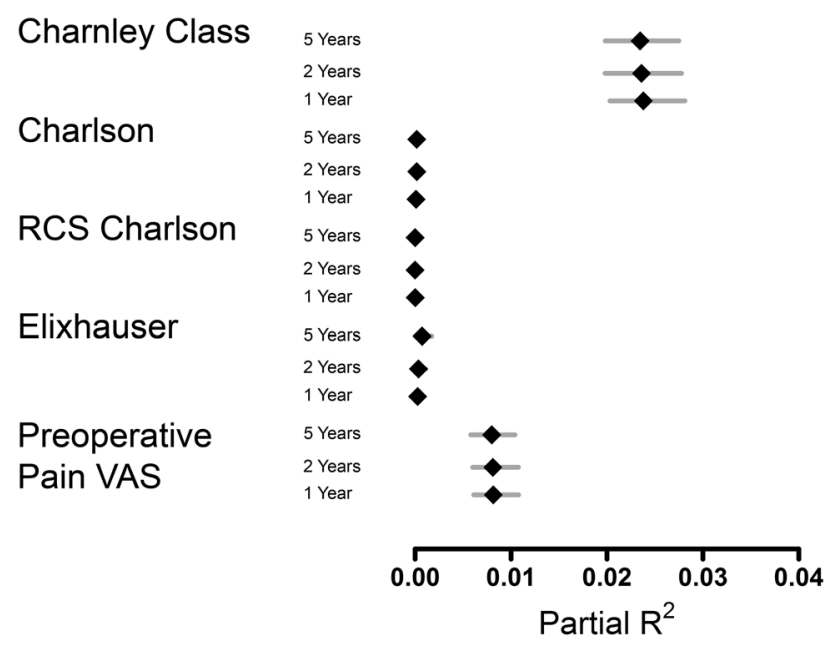

Fig. 4 This forest plot displays the predictive power of ICD-10-based comorbidity measures, self-reported disease-specific comorbidity measure, and the preoperative pain VAS values of the postoperative pain VAS values.

$\mathrm{p}=0.013$ ) (Table 4). For the pain VAS, the predictive power of the 1-year timeframe model was similar to the 2-year timeframe model's predictive power $\left(\mathrm{R}^{2}\right.$ difference $=-0.0004 ; 95 \% \mathrm{CI},-0.001$ to $0.0001 ; \mathrm{p}=0.001$ ). The 5-year timeframe model slightly outperformed both the 1 -year timeframe model $\left(\mathrm{R}^{2}\right.$ difference $=-0.001 ; 95 \% \mathrm{CI}$, -0.0023 to $-0.0004, \mathrm{p}=0.001)$ and the 2 -year timeframe model $\left(\mathrm{R}^{2}\right.$ difference $=-0.0008 ; 95 \% \mathrm{CI},-0.0016$ to $-0.0001 ; \mathrm{p}=0.013$ ) (Table 5). Finally, for the satisfaction VAS, the predictive power of the 1-year timeframe model was significantly lower than the 2-year timeframe model $\left(\mathrm{R}^{2}\right.$ difference $=-0.0012 ; 95 \% \mathrm{CI},-0.002$ to -0.0006 ; $\mathrm{p}=0.001)$ and, once again, the 5-year timeframe model outperformed both the 1-year timeframe model $\left(\mathrm{R}^{2}\right.$ difference $=-0.002 ; 95 \% \mathrm{CI},-0.0036$ to $-0.0012 ; \mathrm{p}=0.001$ ) and the 2-year timeframe model $\left(\mathrm{R}^{2}\right.$ difference $=-0.001$; $95 \% \mathrm{CI},-0.0022$ to $-0.0003 ; \mathrm{p}=0.013$ ) (Table 6). Although these increases were statistically significant, their clinical importance would need to be determined for each outcome measure.

\section{Discussion}

National registry data can help us to better understand PROs. When conducting these analyses, proper risk adjustment is critical to understand how patient comorbidities influence these surgical outcomes after THA. Unfortunately, there is no consensus on how best to incorporate current comorbidity indices in registry-based predictive models. For the purpose of predicting patient HRQoL, pain, and satisfaction 1 year after undergoing THA, we found that there was no added value in the ICD-10-based comorbidity measures if the

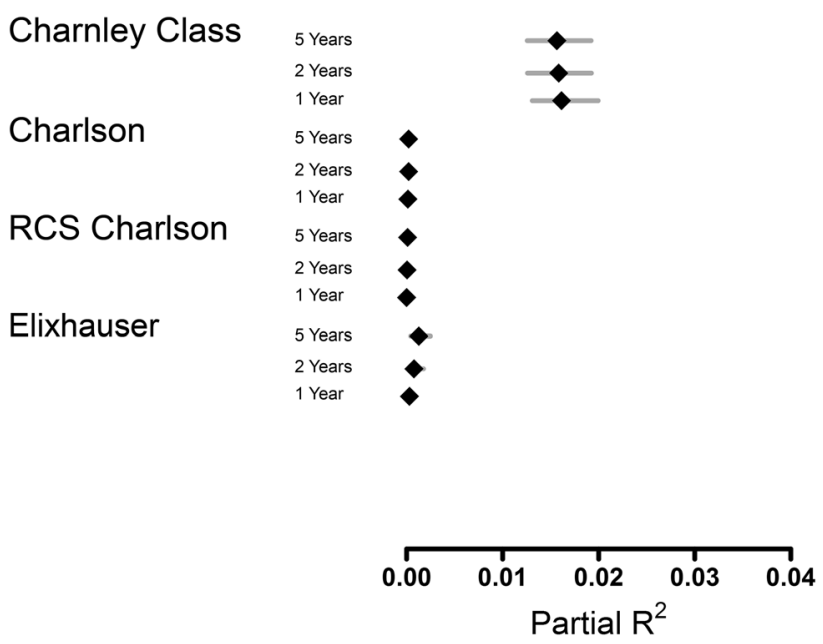

Fig. 5 This forest plot displays the predictive power of ICD-10-based comorbidity measures and self-reported disease-specific comorbidity measure of the postoperative satisfaction VAS values.

patient's Charnley classification and preoperative HRQoL and pain measures were known regardless of the timeframe used to calculate the comorbidity measures. All three comorbidity measures considered (Elixhauser, Charlson, and the RCS Charlson) had low predictive power.

There were a few limitations to our investigation. Sweden has a very successful arthroplasty registry and PROM followup model with $98 \%$ of all THAs captured and a 90\% PROM response rate at 1 year after surgery; however, not all data in our investigation could be obtained from the Swedish Hip Arthroplasty Register. The ICD-10 codes used to identify and calculate the comorbidity measures were obtained from the Swedish National Patient Registry. Unfortunately, the National Patient Registry only collects diagnosis codes of hospitalized patients but not diagnosis codes identified by general practitioners; therefore, some comorbid conditions may have been missed during calculation of the comorbidity measures. ICD-10 codes for general practitioners are gathered at the county level but collection of records from all 21 Swedish counties would have been cost- and time-prohibitive for our project. In addition to the limitation that comorbidities included in this study were restricted to those reported during hospitalizations, the accuracy of reporting all relevant diagnoses at discharge may be questioned, because of the challenges associated with administrative coding. We were also limited to including only patients with complete pre- and postoperative PROs. Although the Swedish Hip Arthroplasty Register had 100\% nationwide coverage of all hospitals during the observation period, only $92 \%$ of the hospitals were participating in the routine PROMs followup by 2007, which meant that some hospitals were not included in our analyses [13]. Finally, an important consideration for these analyses is that THA is an elective 
Table 2. Predictive power of International Classification of Diseases-based comorbidity measures of the postoperative PROM values

\begin{tabular}{|c|c|c|c|c|c|c|}
\hline \multirow[t]{2}{*}{ Scale } & \multicolumn{3}{|l|}{ EQ-5D } & \multicolumn{3}{|l|}{ EQ VAS } \\
\hline & Univariate & Adjusted* & Adjusted $^{\dagger}$ & Univariate & Adjusted* & Adjusted $^{\dagger}$ \\
\hline \multicolumn{7}{|l|}{ Charlson } \\
\hline 1 year & 0.007 & 0.005 & 0.003 & 0.013 & 0.011 & 0.009 \\
\hline 2 years & 0.009 & 0.007 & 0.005 & 0.017 & 0.014 & 0.012 \\
\hline 5 years & 0.011 & 0.009 & 0.006 & 0.020 & 0.016 & 0.014 \\
\hline \multicolumn{7}{|c|}{ RCS Charlson } \\
\hline 1 year & 0.004 & 0.002 & 0.001 & 0.008 & 0.006 & 0.005 \\
\hline 2 years & 0.003 & 0.002 & 0.001 & 0.008 & 0.006 & 0.005 \\
\hline 5 years & 0.003 & 0.002 & 0.001 & 0.008 & 0.006 & 0.005 \\
\hline \multicolumn{7}{|l|}{ Elixhauser } \\
\hline 1 year & 0.006 & 0.005 & 0.003 & 0.011 & 0.008 & 0.007 \\
\hline 2 years & 0.009 & 0.007 & 0.005 & 0.014 & 0.011 & 0.010 \\
\hline 5 years & 0.013 & 0.010 & 0.007 & 0.019 & 0.015 & 0.013 \\
\hline \multirow[t]{2}{*}{ Scale } & \multicolumn{3}{|l|}{ Pain VAS } & \multicolumn{3}{|c|}{ Satisfaction VAS } \\
\hline & Univariate & Adjusted* & Adjusted $^{\dagger}$ & Univariate & Adjusted* & Adjusted $^{\dagger}$ \\
\hline \multicolumn{7}{|l|}{ Charlson } \\
\hline 1 year & 0.002 & 0.001 & 0.001 & 0.001 & 0.001 & - \\
\hline 2 years & 0.002 & 0.001 & 0.001 & 0.002 & 0.001 & - \\
\hline 5 years & 0.002 & 0.002 & 0.002 & 0.002 & 0.002 & - \\
\hline \multicolumn{7}{|c|}{ RCS Charlson } \\
\hline 1 year & 0.001 & 0.001 & 0.000 & 0.001 & 0.000 & - \\
\hline 2 years & 0.001 & 0.001 & 0.000 & 0.001 & 0.000 & - \\
\hline 5 years & 0.001 & 0.000 & 0.000 & 0.000 & 0.000 & - \\
\hline \multicolumn{7}{|c|}{ Elixhauser } \\
\hline 1 year & 0.002 & 0.001 & 0.001 & 0.001 & 0.001 & - \\
\hline 2 years & 0.002 & 0.001 & 0.001 & 0.003 & 0.002 & - \\
\hline 5 years & 0.004 & 0.003 & 0.002 & 0.004 & 0.003 & - \\
\hline
\end{tabular}

* Adjusted for Charnley classification; 'adjusted for Charnley classification and preoperative PROM; PROM = patient-reported outcome measure; VAS = visual analog scale; RCS = Royal College of Surgeons.

Table 3. Influence of measures on postoperative EQ-5D index values

\begin{tabular}{|c|c|c|c|c|c|c|}
\hline \multirow[t]{2}{*}{ Measure } & \multicolumn{2}{|c|}{1 year pre-THA } & \multicolumn{2}{|c|}{2 years pre-THA } & \multicolumn{2}{|c|}{5 years pre-THA } \\
\hline & Coefficient & $95 \%$ CI & Coefficient & $95 \%$ CI & Coefficient & $95 \%$ CI \\
\hline Intercept & 0.785 & $0.778-0.791$ & 0.787 & $0.78-0.794$ & 0.791 & $0.784-0.798$ \\
\hline \multicolumn{7}{|l|}{ Charnley Class } \\
\hline A & Reference & & Reference & & Reference & \\
\hline B & -0.064 & -0.073 to -0.054 & -0.064 & -0.073 to -0.054 & -0.065 & -0.074 to -0.055 \\
\hline $\mathrm{C}$ & -0.112 & -0.119 to -0.105 & -0.111 & -0.118 to -0.105 & -0.111 & -0.117 to -0.104 \\
\hline Charlson* & -0.023 & -0.035 to -0.011 & -0.025 & -0.034 to -0.015 & -0.02 & -0.027 to -0.012 \\
\hline RCS Charlson* & 0.003 & -0.008 to 0.015 & 0.009 & -0.001 to 0.02 & 0.01 & $0.001-0.019$ \\
\hline Elixhauser* & -0.010 & -0.015 to -0.004 & -0.012 & -0.017 to -0.007 & -0.015 & -0.019 to -0.01 \\
\hline Preoperative value & 0.147 & $0.137-0.156$ & 0.146 & $0.136-0.155$ & 0.145 & $0.135-0.154$ \\
\hline $\mathrm{R}^{2}$ & 0.107 & & 0.109 & & 0.112 & \\
\hline
\end{tabular}

* Calculated based on patient diagnoses from overlapping preoperative time periods of 1,2 , and 5 years; $\mathrm{CI}=$ confidence interval; RCS $=$ Royal College of Surgeons. 
Table 4. Influence of measures on patient postoperative EQ VAS values

\begin{tabular}{|c|c|c|c|c|c|c|}
\hline \multirow[t]{2}{*}{ Measure } & \multicolumn{2}{|c|}{1 year pre-THA } & \multicolumn{2}{|c|}{2 years pre-THA } & \multicolumn{2}{|c|}{5 years pre-THA } \\
\hline & Coefficient & $95 \% \mathrm{CI}$ & Coefficient & $95 \% \mathrm{CI}$ & Coefficient & $95 \% \mathrm{CI}$ \\
\hline Intercept & 71.727 & $70.938-72.516$ & 71.932 & $71.143-72.720$ & 72.347 & $71.555-73.14$ \\
\hline \multicolumn{7}{|l|}{ Charnley Class } \\
\hline A & Reference & & Reference & & Reference & \\
\hline B & -4.392 & -5.196 to -3.588 & -4.398 & -5.201 to -3.596 & -4.485 & -5.286 to -3.684 \\
\hline $\mathrm{C}$ & -8.95 & -9.509 to -8.391 & -8.872 & -9.43 to -8.314 & -8.83 & -9.387 to -8.273 \\
\hline Charlson* & -3.407 & -4.400 to -2.414 & -3.429 & -4.249 to -2.608 & -2.638 & -3.294 to -1.982 \\
\hline RCS Charlson* & -0.016 & -1.012 to 0.980 & 0.373 & -0.539 to 1.286 & 0.244 & -0.592 to 1.079 \\
\hline Elixhauser* & -0.886 & -1.353 to -0.420 & -1.054 & -1.49 to -0.619 & -1.332 & -1.723 to -0.94 \\
\hline Preoperative value & 0.174 & $0.162-0.186$ & 0.174 & $0.162-0.186$ & 0.173 & $0.161-0.184$ \\
\hline $\mathrm{R}^{2}$ & 0.108 & & 0.111 & & 0.115 & \\
\hline
\end{tabular}

* Calculated based on diagnoses from overlapping preoperative time periods of 1, 2, and 5 years; VAS $=$ visual analog scale; CI $=$ confidence interval; RCS $=$ Royal College of Surgeons.

Table 5. Influence of measures on postoperative pain VAS values

\begin{tabular}{|c|c|c|c|c|c|c|}
\hline \multirow[t]{2}{*}{ Measure } & \multicolumn{2}{|c|}{1 year pre-THA } & \multicolumn{2}{|c|}{2 years pre-THA } & \multicolumn{2}{|c|}{5 years pre-THA } \\
\hline & Coefficient & $95 \% \mathrm{CI}$ & Coefficient & $95 \% \mathrm{CI}$ & Coefficient & $95 \% \mathrm{CI}$ \\
\hline Intercept & 4.752 & $3.83-5.674$ & 4.699 & $3.777-5.622$ & 4.588 & $3.666-5.51$ \\
\hline \multicolumn{7}{|l|}{ Charnley Class } \\
\hline A & Reference & & Reference & & Reference & \\
\hline B & 4.187 & $3.444-4.929$ & 4.189 & $3.447-4.932$ & 4.224 & $3.481-4.967$ \\
\hline $\mathrm{C}$ & 5.848 & $5.335-6.36$ & 5.823 & $5.31-6.335$ & 5.799 & $5.287-6.311$ \\
\hline Charlson* & 0.688 & -0.23 to 1.607 & 0.752 & -0.009 to 1.512 & 0.596 & -0.013 to 1.204 \\
\hline RCS Charlson* & 0.245 & -0.675 to 1.166 & 0.094 & -0.751 to 0.939 & -0.061 & -0.836 to 0.714 \\
\hline Elixhauser* & 0.522 & $0.091-0.954$ & 0.569 & $0.167-0.972$ & 0.732 & $0.369-1.096$ \\
\hline Preoperative value & 0.096 & $0.082-0.111$ & 0.096 & $0.082-0.110$ & 0.096 & $0.081-0.110$ \\
\hline $\mathrm{R}^{2}$ & 0.037 & & 0.037 & & 0.038 & \\
\hline
\end{tabular}

* Charlson, RCS Charlson, and the Elixhauser were calculated based on diagnoses from overlapping time periods of 1, 2, and 5 years; VAS = visual analog scale; $\mathrm{CI}=$ confidence interval; RCS $=$ Royal College of Surgeons.

procedure, which fundamentally limits the population for whom this treatment is available. Patients must be healthy enough to be eligible to undergo THA, as evidenced by the comorbidity indices in this population, and therefore, comorbidity measures aimed at investigating mortality are inherently unlikely to be associated with how a patient will report their HRQoL, pain, or satisfaction after the procedure.

We also acknowledge that gender and age are important confounders influencing the studied comorbidity measures and PROMs. Moreover, in this setting, the comorbidity measures (Charnley classification and the ICD-10-based comorbidity measures) act as mediators that channel some of the effects of age and gender on PROMs. We opted not to present results adjusted for age and gender to see the maximal predictive power that the comorbidity measures had on PROMs. Additionally, models regressing postoperative PROMs on gender and age, preoperative PROMs, Charnley classification, and the ICD-10-based comorbidity measures (together or one at the time) showed that neither age nor gender modified the effect or predictive power of the Charnley classification and the ICD-10-based comorbidity measures. Both age and gender had predictive power lower than $1 \%$.

It is important to remember that the self-administered Charnley classification survey was used in our investigation. Despite the simplicity of the original Charnley classification [5], surgeon-assigned classification has a high 
Table 6. Influence of measures on postoperative satisfaction VAS values

\begin{tabular}{|c|c|c|c|c|c|c|}
\hline \multirow[t]{2}{*}{ Measure } & \multicolumn{2}{|c|}{1 year pre-THA } & \multicolumn{2}{|c|}{2 years pre-THA } & \multicolumn{2}{|c|}{5 years pre-THA } \\
\hline & Coefficient & $95 \% \mathrm{CI}$ & Coefficient & $95 \% \mathrm{CI}$ & Coefficient & $95 \% \mathrm{CI}$ \\
\hline Intercept & 13.243 & $12.82-13.667$ & 13.09 & $12.664-13.515$ & 12.895 & $12.465-13.325$ \\
\hline \multicolumn{7}{|l|}{ Charnley Class } \\
\hline A & Reference & & Reference & & Reference & \\
\hline B & 2.959 & $2.120-3.797$ & 2.964 & $2.126-3.802$ & 3.009 & $2.171-3.847$ \\
\hline $\mathrm{C}$ & 5.431 & $4.854-6.007$ & 5.384 & $4.807-5.96$ & 5.349 & $4.773-5.925$ \\
\hline Charlson* & 0.88 & -0.158 to 1.918 & 0.908 & $0.049-1.767$ & 0.742 & $0.054-1.429$ \\
\hline RCS Charlson* & -0.074 & -1.115 to 0.967 & -0.505 & -1.46 to 0.45 & -0.658 & -1.533 to 0.218 \\
\hline Elixhauser* & 0.628 & $0.141-1.116$ & 0.936 & $0.481-1.391$ & 1.087 & $0.676-1.497$ \\
\hline $\mathrm{R}^{2}$ & 0.018 & & 0.019 & & 0.020 & \\
\hline
\end{tabular}

* Charlson, RCS Charlson, and the Elixhauser were calculated based on diagnoses from overlapping time periods of 1, 2, and 5 years; VAS $=$ visual analog scale; $\mathrm{CI}=$ confidence interval; $\mathrm{RCS}=$ Royal College of Surgeons .

rate of interobserver variability [2]. Charnley Class $\mathrm{C}$, as reported by McGuigan et al., was approximately 4\% [16], whereas Lavernia's group reported 30\% [14]. Complicating matters further, some researchers have split Class B into two groups: those who have not been operated on the contralateral hip (B1) and those who have (B2) [7, 21]. It was recently shown that the classification does not require splitting because patients naturally classify themselves similarly to Charnley classification definitions [10]. For these reasons, clinicians are encouraged to use the selfadministered Charnley classification survey rather than assign the classification themselves.

Charnley classification and the preoperative value of the outcome examined contributed most to the models' predictive power rather than the three comorbidity measures tested. Similarly, Lavernia et al. [14] found comorbidity scores correlated poorly with patient pain level and functional abilities after total joint arthroplasty. Interestingly, Charnley classification had stronger predictive power than the patient preoperative health state for the EQ-5D index, EQ VAS, and, most evidently, for the postoperative pain VAS. Röder et al. [20] drew similar conclusions, finding that patients with poor preoperative walking capacity and hip flexion were more likely to have poorer clinical results after THA. In contrast to our findings, however, Röder et al. found no association between patient preoperative pain level and pain alleviation. Of the three comorbidity measures investigated, Elixhauser comorbidity scores were most predictive and RCS Charlson scores the least. Based on an extensive review, Sharabiana et al. [23] found that if a comorbidity measure were necessary, the Elixhauser comorbidity score might be the best choice, but they had reservations that the results might differ based on the patient group and the outcome being investigated. However, one must consider that predictive performance of all the comorbidity index scores was not only outcome; the risk-adjustment method also had bearing [17], as was seen in patients with osteoporosis $[6,15]$. Based on our results, we conclude that if consideration of patient comorbidities is necessary to achieve a particular purpose, then the Elixhauser comorbidity score would seem the best for predicting patient HRQoL, pain, and satisfaction 1 year after THA. It is important to remember that although the comorbidity indices we used had low predictive capacity with regard to the PROMs explored, Mnatzaganian et al. [17] proved the index scores to be valuable for predicting patient in-hospital mortality, adverse events, and nonroutine discharge after major orthopaedic surgery. Furthermore, our results indicated that regardless of comorbid conditions covered by the Charlson and Elixhauser indices, patients with similar self-reported preoperative HRQoL and pain as well as similar demography have similar chances to improve from surgery.

We observed a small increase in predictive power with the increase of preoperative timeframe for our study; however, despite statistical significance, the increase lacked practical importance, suggesting that comorbidities gathered 1 year before surgery were nearly as useful as those gathered much earlier. Similarly, when examining the influence of the same comorbidity indices on rates of THA reoperation, Gordon et al. found that there was little added value by extending the timeframe beyond 1 year [11]. For these reasons, it seems unnecessary to try to obtain information about comorbid conditions more than 1 year before THA if one were to include a comorbidity index as a risk adjustment for predicting outcomes after surgery.

Given the observational nature of registry studies, proper risk adjustment is critical to eliminate confounding when exploring PRO after treatment. National arthroplasty registry data provide a great way to explore the influence of demographic, surgical, and preoperative health states on outcomes after THA. The comorbidity measures explored 
in our analyses were originally designed to identify rates of patient mortality and hospitalization [4, 8, 23]. It may be for that reason that only weak associations were found between the measures and PRO after THA. Identification of patient-reported Charnley classification appears to be important in understanding how a patient will fare after hip arthroplasty. A patient's Charnley classification could be an important risk-adjustment tool, which may be considered by the clinician as well as by researchers for predicting patient HRQoL, pain, and satisfaction after THA.

\section{References}

1. Ahmad MA, Xypnitos FN, Giannoudis PV. Measuring hip outcomes: common scales and checklists. Injury Int J Care Inj. 2011;42:259-264.

2. Bjorgul K, Novicoff WM, Saleh KJ. Evaluating comorbidities in total hip and knee arthroplasty: available instruments. J Orthop Traumatol. 2010;11:203-209.

3. Callaghan JJ, Dysart SH, Savory CF, Hopkinson WJ. Assessing the results of hip replacement. A comparison of five different rating systems. J Bone Joint Surg Br. 1990;72:1008-1009.

4. Charlson ME, Pompei P, Ales KL, MacKenzie CR. A new method of classifying prognostic comorbidity in longitudinal studies: development and validation. J Chronic Dis. 1987;40: 373-383.

5. Charnley J. The long-term results of low-friction arthroplasty of the hip performed as a primary intervention. J Bone Joint Surg Br. 1972;54:61-76.

6. Dominick KL, Dudley TK, Coffman CJ, Bosworth HB. Comparison of three comorbidity measures for predicting health service use in patients with osteoarthritis. Arthritis Rheum Arthritis Care Res. 2005;53:666-672.

7. Dunbar MJ, Robertsson O, Ryd L. What's all that noise? The effect of co-morbidity on health outcome questionnaire results after knee arthroplasty. Acta Orthop Scand. 2004;75:119-126.

8. Elixhauser A, Steiner C, Harris DR, Coffey RM. Comorbidity measures for use with administrative data. Med Care. 1998; 36:8-27.

9. EuroQolGroup. EuroQol - a new facility for the measurement of health related quality of life. Health Policy. 1990;16:199-208.

10. Gordon M, Frumento P, Skoldenberg O, Greene M, Garellick G, Rolfson $\mathrm{O}$. Women in Charnley class $\mathrm{C}$ fail to improve in mobility to a higher degree after total hip replacement. Acta Orthop. 2014;85:335-341.

11. Gordon M, Stark A, Skoldenberg OG, Karrholm J, Garellick G. The influence of comorbidity scores on re-operations following primary total hip replacement: comparison and validation of three comorbidity measures. Bone Joint J. 2013;95:1184-1191.

12. Greene ME, Rolfson O, Nemes S, Gordon M, Malchau H, Garellick G. Education attainment is associated with patientreported outcomes: findings from the Swedish Hip Arthroplasty Register. Clin Orthop Relat Res. 2014;472:1868-1876.

13. Karrholm J, Garellick G, Rogmark C, Herberts P. Swedish Hip Arthroplasty Register Annual Report 2007. Gothenburg, Sweden: Swedish Hip Arthroplasty Register; 2008.

14. Lavernia CJ, Laoruengthana A, Contreras JS, Rossi MD. Allpatient refined diagnosis-related groups in primary arthroplasty. J Arthroplasty. 2009;24:19-23.

15. Lix LM, Quail J, Teare G, Acan B. Performance of comorbidity measures for predicting outcomes in population-based osteoporosis cohorts. Osteoporosis Int. 2011;22:2633-2643.

16. McGuigan FX, Hozack WJ, Moriarty L, Eng K, Rothman RH. Predicting quality-of-life outcomes following total joint arthroplasty. Limitations of the SF-36 Health Status Questionnaire. J Arthroplasty. 1995;10:742-747.

17. Mnatzaganian G, Ryan P, Hiller JE. Does co-morbidity provide significant improvement on age adjustment when predicting medical outcomes? Methods Inf Med. 2014;53:115-120.

18. Mnatzaganian G, Ryan P, Norman PE, Hiller JE. Accuracy of hospital morbidity data and the performance of comorbidity scores as predictors of mortality. J Clin Epidemiol. 2012;65: $107-115$

19. Quan H, Sundararajan V, Halfon P, Fong A, Burnand B, Luthi J-C, Saunders LD, Beck CA, Feasby TE, Ghali WA. Coding algorithms for defining comorbidities in ICD-9-CM and ICD-10 administrative data. Med Care. 2005;43:1130-1139.

20. Röder C, Staub LP, Eggli S, Dietrich D, Busato A, Muller U. Influence of preoperative functional status on outcome after total hip arthroplasty. J Bone Joint Surg Am. 2007;89:11-17.

21. Röder C, Staub LP, Eichler P, Widmer M, Dietrich D, Eggli S, Muller U. Avoiding misclassification bias with the traditional Charnley classification: rationale for a fourth Charnley class BB. J Orthop Res. 2006;24:1803-1808.

22. Rolfson O, Karrholm J, Dahlberg LE, Garellick G. Patientreported outcomes in the Swedish Hip Arthroplasty register: results of a nationwide prospective observational study. $J$ Bone Joint Surg Br. 2011;93:867-875.

23. Sharabiani MTA, Aylin P, Bottle A. Systematic review of comorbidity indices for administrative data. Med Care. 2012;50: $1109-1118$. 